Voix et Images

voixetimages

\title{
Le double et son théâtre
}

\section{Lucie Robert}

Volume 15, numéro 2 (44), hiver 1990

Pratiques illicites

URI : https://id.erudit.org/iderudit/200848ar

DOI : https://doi.org/10.7202/200848ar

Aller au sommaire du numéro

\section{Éditeur(s)}

Université du Québec à Montréal

\section{ISSN}

0318-9201 (imprimé)

1705-933X (numérique)

Découvrir la revue

\section{Citer cet article}

Robert, L. (1990). Le double et son théâtre. Voix et Images, 15(2), 310-314.

https://doi.org/10.7202/200848ar d'utilisation que vous pouvez consulter en ligne.

https://apropos.erudit.org/fr/usagers/politique-dutilisation/ 


\section{Dramaturgie}

\section{Le double et son théâtre}

\section{par Lucie Robert, Université du Québec à Montréal}

Depuis sa fondation en 1984, l'Association québécoise des critiques de théâtre a montré un grand dynamisme, notamment en créant sa série de prix de théâtre, remis chaque année le jour de la Sainte Fleur (le 5 octobre). Linitiative la plus récente de l'association est la réunion de treize textes, écrits par autant de critiques qui cherchent à faire le bilan de la saison théâtrale 1988-1989. Veilleurs de nuit. Saison théâtrale $1988-1989^{1}$ est publié sous la direction de Gilbert David avec une présentation de Michel Vaïs, président de l'A.Q.C.T.

Lobjectif premier de cette publication est de diffuser la réflexion des critiques de théâtre. Ils et elles s'entendent ainsi pour déplorer le peu de recherche et de création théâtrale chez les troupes en particulier celles que l'on dit «institutionnelles ". Ils et elles s'entendent également pour saluer le talent des acteurs et des actrices et pour regretter le faible intérêt des textes dramatiques originaux, écrits par des auteurs qui, par ailleurs, ne paraissent pas trouver de metteurs en scène capables de leur rendre justice. On sait d'ailleurs la réaction vive qu'a suscitée chez les dramaturges québécois la décision de l'A.Q.C.T de ne pas décerner en 1989 le prix de la meilleure pièce, le jury ayant été incapable de s'entendre. On trouve également en annexe la liste des spectacles présentés à Montréal (on nous promet celles de Québec et de Hull-Ottawa pour la prochaine édition), la programmation du troisième Festival des Amériques. Les responsables profitent par ailleurs de l'occasion pour faire connaître l'Association québécoise des critiques de théâtre dont le code d'éthique et de déontologie est reproduit en annexe.

Un tel recueil d'articles pose le problème d'une esthétique où la critique et la pratique théâtrale sont soumises aux mêmes normes institutionnelles et, comme l'ont montré Jean-Claude Germain, Benoît Laplante et Pierre Lavoie ${ }^{2}$, s'adressent au même public. Selon le lieu de travail (journal, radio, télévision, revue culturelle, enseignement) on pratique une critique plus ou moins esthétique, plus ou moins moderne, plus libre dans l'ensemble que celle que des praticiens et des praticiennes qui ont une salle à remplir. Aussi Veilleurs de nuit témoigne-t-il d'une quête et d'une recherche qui avance plus vite vers la théâtralité et vers la modernité que celle des praticiens et des praticiennes et qui par conséquent est condamnée à l'insatisfaction permanente, confirmant ainsi le lieu commun qui veut que les artistes et les critiques forment en réalité un vieux couple, insatisfait de sa liaison, mais inséparable. Le recueil pose également le problème de son destinataire. Les critiques de théâtre écrivent normalement pour le public de théâtre, qui cherche dans la critique une sorte de publicité informative. Veilleurs de nuit ne peut, par sa composition même, que s'adresser aux gens de théâtre, comme un avertissement, et aux critiques, comme une pratique didentification collective. Il en résulte une forme de narcissisme, une "représentation" de la critique si l'on veut, plus évidente dans certains textes que dans d'autres, qui 
agace à la longue et qui n'est compensée que par l'évidente passion qui habite chacun, chacune des signataires.

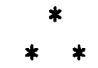

Une réflexion semblable habite la pièce de Bernard Andrès, la Doublure ${ }^{3}$, pièce non jouée qui sera sans doute la dernière publiée par Guérin Littérature. Écrite par un professeur qui est aussi un critique et un romancier, la pièce est une allégorie sur le théâtre et son double, selon Artaud, et elle pose une série de questions sur la relation entre un auteur et ses personnages, entre un metteur en scène et les comédiens, entre les comédiens et leurs personnages. Jeu sur le double donc, qui trouve sa forme dans un double texte, à la fois théâtre dans le théâtre et théâtre sur le théâtre. La première partie met en scène trois personnages, le Maitre, le Fou et Sarah, qui préparent à des titres divers la prise du pouvoir dans un État quelconque après l'Alerte. La seconde partie met en scène les trois comédiens Raymond, Michel et Diane préparant la première de la pièce qu'ils doivent jouer le soir même. La seconde partie se trouve donc à théâtraliser la première, c'est-à-dire à rendre apparent le fait que nous sommes plutôt deux fois qu'une au théâtre.

La Doublure est ainsi une pièce savante, "achevée" dit la couverture. Le texte dense, massif, laisse peu de place à la scène et encore moins à l'imagination des lecteurs et des lectrices. L'action est très expliquée. L'auteur en effet nous présente son analyse en même temps que son texte. La didascalie ajoute aux principes de répétition qui construisent le texte: répétition de certaines scènes et dialogues, dédoublement des personnages et des comédiens, présence de jumeaux, mises en abyme, citations et explications. Quoique intéressant, le texte présente certains défauts fréquents chez les romanciers dramaturges qui tendent à combler les vides du texte (comme dans le roman) plutôt à les laisser à l'imagination d'une mise en scène ou d'une lecture. Ainsi, à un certain moment, le Maître annonce: Ce soir, ce sera leur fête [... .] Et je tirerai les ficelles. L'auteur fait la même chose alors qu'il devrait donner de la corde, jouer le rôle du fou plutôt que celui du maître.

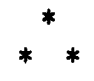

Créée le 5 février 1987 par le théâtre du Double Signe à Sherbrooke, Caryopse ou le Monde entier ${ }^{4}$ de Laurence Tardi est publié, sans raison apparente, dans la revue les Herbes rouges, plutôt que dans la collection "Théâtre" de la maison. Histoire de double encore une fois, la pièce est une réflexion sur la création dramatique: un personnage désigné par sa fonction, l'Auteure, y crée un personnage, Joël, qui lui sert à réaliser une sorte d'exorcisme, une libération de sa vie antérieure et de ses angoisses. Au dédoublement du personnage correspond le dédoublement de l'espace, et une superposition de temps divers, celui de la créatrice, celui du personnage, puis, dans une dimension 
supplémentaire, le passé de chacune. Les personnages se mirent ainsi l'une dans l'autre, jusqu'au rapport amoureux, redoublant cette fois la relation amoureuse sans espoir que l'auteure a entretenue autrefois avec une autre femme, Leslie. Ultimement, l'on atteindra la fusion des doubles de manière à reconstituer le caryopse, fruit qui ne s'ouvre pas et dont la graine unique est enveloppée, image de la vie intime, privée, essentielle. Aussi la présence du troisième personnage, l'Homme, représente-t-elle l'intrusion d'un monde extérieur hostile qui tend à déposséder la femme de son monde à elle, le monde entier du titre, en brisant le parfait équilibre créé par cette structure en miroir. Le texte est intéressant quoique ici encore, le narcissisme de la réflexion à la fois dramaturgique et existentielle prenne le dessus sur la relation dialogique avec le public.

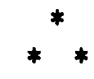

Alberto Kurapel est un chilien qui vit au Québec depuis 1973. Son Prométhée enchaîné ${ }^{5}$ est présenté doublement, dans une édition bilingue français-espagnol qui reproduit de cette manière la production scénique, bilingue elle aussi, présentée à l'Espace Exilio par la compagnie des Arts Exilio, le 11 mars 1988. Le texte est traduit phrase à phrase, sans coupure typographique, pour mimer la représentation où les mêmes phrases étaient dites à la suite, sans pause. Il en résulte une édition dont la lecture est pour le moins épuisante, les deux textes étant à la suite plutôt qu'en regard, demandant ainsi que l'on s'ajuste rapidement au changement dans les codes linguistiques. Le texte est accompagné d'une note biographique, d'une préface, d'une postface, d'une bibliographie, d'une revue de presse, tout aussi bilingues. Ces textes d'appoint et les photographies de scène de George Smid font près de la moitié du volume.

En réalité, le Prométhée enchaîné d'Alberto Kurapel est une réflexion sur l'espace. Le théâtre Exilio habite une salle assez particulière, à la périphérie du centre montréalais, où il arrive fréquemment que l'on intègre à la représentation les trains qui passent à proximité. L'espace est ainsi tout autant sonore que visuel, ce que confirme la superposition des langues nationales. De même, Kurapel est connu d'abord pour ses performances. L'étrangeté du texte publié est liée à cet état de fait. À partir du mythe, qui met en cause l'autorité, l'auteur pense la situation du Chili. Le travail gestuel et spatial inscrivent cette réflexion dans les formes les plus américaines du kitsch contemporain. Le résultat est indéfinissable.

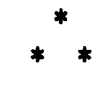

Dans la collection qu'elle dirige aux éditions Trois, Anne-Marie Alonzo publie une pièce de Gloria Escomel, présentée en 1979 à la radio MF de Radio-Canada dans une réalisation de Madeleine Gérôme, à l'émission Premières. Tu en parleras ... et après ? 6 a déjà connu une longue et fructueuse carrière, méritant un prix des Radios européennes en 1980. Elle a été diffusée en Suisse, au Japon, 
en Norvège et en Yougoslavie, entre 1980-1988. Dédiée aux membres d'Amnistie international, d̀ celles et ceux qui travaillent au rétablissement et au maintien des droits de la personne partout dans le monde, la pièce annonce clairement ses couleurs et son sujet: la torture. L'écriture dramatique crée deux plans sonores: au premier, on entend une discussion entre prisonniers, essentiellement une avocate et un journaliste, à l'arrière-plan des cris et des tango. La pièce ne cache pas sa thèse et n'entend pas se perdre dans des subtilités esthétiques, quoique dans l'ensemble elle soit assez bien conçue. L'efficacité de l'ensemble est assez remarquable tout en demeurant très économique de moyens.

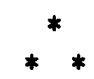

Encore aux éditions Trois, Anne-Marie Alonzo publie la Passion du jeu?, sous-titré "livre-thêâtre et écrit par 54 comédiens et comédiennes du Québec. Louvrage est bien fait dans le genre, les illustrations, des photos de scène pour la plupart, sont bien choisies. La passion que cherche à décrire l'ouvrage est bien belle, mais les comédiennes et les comédiens sont des gens qui manipulent peu et mal le langage, leur instrument étant le corps. Aussi doit-on accepter que les textes présentés soient inégaux dans leur qualité et dans l'intérêt qu'ils offrent. Plus de la moitié des collaborateurs et des collaboratrices dépensent plusieurs pages à expliquer qu'ils et elles ne peuvent décrire ni expliquer cet intérêt ou cette passion. Plusieurs ont recours à une notice autobiographique ou à une comparaison avec les jeux d'enfants. Il résulte de l'ensemble une sorte de narcissisme, de naïveté inévitable dans le genre, avec un ensemble de clichés et de lieux communs prévisibles, d'autant que ceux et celles qui n'ont pas répondu à l'invitation d'Anne-Marie Alonzo sont précisément ceux et celles qui auraient pu apporter un témoignage inédit, soit sur la difficulté du métier, soit sur son impossibilité. Le livre est beau, mais il n'apporte rien de vraiment neuf à notre connaissance ou à notre appréciation de ce "divin" métier.

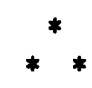

En terminant, je voudrais signaler en particulier le dossier consacré par la revue Jeu ${ }^{8}$ à la pièce $A$ quelle heure on meurt? relecture de l'œuvre de Réjean Ducharme par Martin Faucher, concepteur du spectacle qui mettait en scène les personnages de Mille Milles et de Chateaugué. Les trois articles qui composent le dossier sont de Louise Vigeant (du roman à la scène), Solange Lévesque (de la scène à la salle) et Jean-Francois Chassay (du lecteur à l'écrivain). L'essentiel du numéro porte toutefois sur la marionnette. On notera les articles de Michel Fréchette sur l'histoire de cette pratique théâtrale particulière au Québec.

1 En collaboration, Veilleurs de nuit. Saison théâtrale 1988-1089, sous la direction de Gilbert David, Montréal, les Herbes rouges, 1989,79 p.

2 Jean-Claude Germain, a Lagent double s, Jeu, $3^{\bullet}$ trimestre 1986, p. 81-88; Benoit Laplante avec la collaboration de Pierre Lavoie, «La critique et son public: enquêtes», ibid., p. 94-110. 
3 Bernard Andrès, la Doublure. Théâtre, Montréal, Guérin tittérature, 1988, 99 p.

4 Laurence tardi, Caryopse ou le Monde entier, Montréal, les Herbes rouges, n 173, 1989, $47 \mathrm{p}$.

5 Alberto Kurapel, Prométhée enchaîné selon Alberto Kurapel le guanaco gaucho. Prometeo encadenado segun Alberto Kurapel le Guanaco gaucho, Montréal, Humanitas nouvelle optique, $1989,114 \mathrm{p}$.

6 Gloria Escomel, Tu en parleras... et après? Théâtre, Montréal, Trois, 1989, 64 p. (Topaze).

7 En collaboration, la Passion du jeu. Livre-théâtre concu par 54 comédiens et comédiennes du Québec, sous la direction d'Anne-Marie Alonzo, Trois, 1989, 238 p. (Passioñ).

8 Jeu, $n^{\circ}$ 51, juin 1989, 216 p. 